\title{
Delirium: The 21st century health care challenge for bedside clinicians
}

\author{
Ruth Richards *' Rejena Azad, Mobolaji Adeola, Betty Clark \\ Houston Methodist Hospital, Houston, United States
}

Received: July 14, 2015

DOI: $10.5430 /$ jnep.v6n6p8
Accepted: November 30, 2015 Online Published: January 20, 2016

URL: http://dx.doi.org/10.5430/jnep.v6n6p8

\begin{abstract}
Delirium is a leading cause of preventable injury in hospitalized patients. Early recognition and intervention for delirium are critical to prevent morbidity and mortality, especially in the older population. Older patients are at increased risk for delirium owing to a combination of age-related changes and environmental factors. Health care providers, including nurses and physicians, often miss delirium symptoms and diagnosis in patients. Without early recognition and treatment, delirium can have significant life-changing consequences in our most vulnerable patients. This acute change in cognition can continue throughout the hospital course and may require additional rehabilitation or placement, delaying transition to home. As the baby boomers age, the older population is expected to increase, with significant implications for health care. With this in mind, the health care team, including frontline caregivers, need to be well informed about delirium. This article will expand readers' knowledge and familiarity with delirium with the purpose of improving their practice and care of the older patient. It will also address the impact of delirium and discuss tools that can help to improve recognition. The most recent advances and current treatment methods to integrate into daily patient care are also discussed. This article places heavy emphasis on identification and prevention of delirium as these are the most important aspect of understanding delirium. Thus, treatment and management are both discussed after prevention since the primary focus of delirium is understanding and preventing this devastating syndrome in our hospitalized patients.
\end{abstract}

Key Words: Delirium, Confusion, Geriatric hospitalized patients

\section{INTRODUCTION}

Extensive research on delirium has been conducted since the 1980s and different investigators have used similar but distinct categories and subcategories to explain the condition. A full understanding of delirium requires consideration of the patient population, causes, environment, prevention, detection, treatment, and outcomes.

The syndrome that we now recognize as delirium has been referred to since the days of Hippocrates, and it was called phrenitis throughout the centuries. ${ }^{[1]}$ The term phrenitis, which means inflammation of the mind, was used to describe delirium up to and through the 18th century. ${ }^{[2]}$ From the 18th century through the late 20th century, interest was sporadic in what was termed "acute confusional state". During the 1980 s, increased interest and research into delirium was preceded by publication of the Diagnostic and Statistical Manual of Mental Disorders, third edition (DSM-III). This edition established delirium as a diagnosis, replacing the term "acute confusional state". [3]

Inouye introduced the Confusion Assessment Method (CAM) to the medical community in 1990, and the ability to accurately diagnose delirium became a reality. The CAM combined 9 criteria of delirium from the Diagnostic and Statistical Manual of Mental Disorders, third edition (DSM-

*Correspondence: Ruth Richards; Email: RWRichards@ @oustonmethodist.org; Address: Houston Methodist Hospital, Houston, United States. 
III-R) into an easy, quick, and reliable four-question tool that any bedside clinician could use. Throughout the 1990s, the CAM was found to be the most reliable and accurate tool for diagnosing delirium. ${ }^{[4]}$ Since 1990 clinicians have continued to research prediction, prevention, intervention, treatment, and outcomes regarding delirium.

Delirium is common, however, it often goes unrecognized in hospitalized older adults. It is defined as an acute change in global cognitive function characterized by inattention and a fluctuating course. The pathophysiology of delirium is poorly understood, because it involves a complex interaction between heterogeneous predisposing and precipitating risk factors. ${ }^{[5]}$ Several theories have been postulated, including inflammatory processes, neurotransmitter imbalances, and physiologic stress. ${ }^{[5,6]}$ Advanced age is an independent predictor, and this is of particular concern because more than $48 \%$ of hospital days are incurred by patients aged 65 years and older. ${ }^{[7]}$ Delirium complicates hospital stays for about 2.3 million older patients and the economic burden is substantial, comparable to that for conditions such as hip fracture, diabetes, and cardiovascular disease. ${ }^{[8]}$

Early recognition of patients at high risk of delirium facilitates immediate intervention and improves prognosis. Routine screening is recommended and feasible in clinical practice. ${ }^{[9]}$ Management strategies are focused on prevention because this has been shown to save lives. Evidence suggests that a multicomponent intervention plan targeting known modifiable risk factors may reduce the incidence of delirium and its adverse outcomes. ${ }^{[10]}$

Due to its significant clinical and economic implications, delirium is emerging as an increasingly important piece of this decade's complex health care puzzle. As such, bedside clinicians must be equipped to effectively manage delirium to improve quality of care for patients. This article will summarize current approaches to the recognition, prevention, and management of delirium in hospitalized older adults.

\section{EPIDEMIOLOGY AND PROGNOSIS}

Delirium is a leading complication in hospitalized older adults. Among acute care populations, approximately $14 \%$ to $24 \%$ of older patients will be admitted with this condition and $6 \%$ to $56 \%$ will develop delirium during hospitalization. $^{[1]}$ An exponential increase in rates of occurrence is observed in more specialized populations such as intensive care, postoperative, and palliative care populations. ${ }^{[5,11-13]}$ Delirium is prevalent in $1.1 \%$ of community-dwelling adults aged 55 years and over, and this prevalence rate increases to $14 \%$ in those over 85 years of age. ${ }^{[14]}$ In nursing homes and post-acute care facilities, up to $60 \%$ of older adults will expe-

Published by Sciedu Press rience delirium. ${ }^{[5]}$ The wide disparity in reported incidence and prevalence rates is due in part to variations in screening instruments and patient populations studied.

Beyond the problems with confusion and agitation, delirium is an independent predictor of short- and long-term adverse health outcomes, even after adjustment for age, illness severity, dementia, and functional status. ${ }^{[15]}$ It has been linked with increased nursing time per patient, prolonged length of hospital stay, and increased morbidity and mortality rates of $25 \%$ to $33 \% .^{[16,17]}$ Hospital costs attributable to delirium are estimated at US $\$ 2,500$ per patient, totaling about $\$ 6.9$ billion (2004 figures) of Medicare hospital expenditures. Additional costs accrue after discharge because of the greater need for long-term care or additional home health care, rehabilitation services, and informal caregiving. ${ }^{[5,17]}$ Leslie and colleagues reported that patients who developed delirium during hospitalization had a $62 \%$ increased risk of mortality within 1 year after discharge. ${ }^{[18]}$ Moreover, long-term consequences such as cognitive and functional decline, which impact a patient's quality of life, cannot be underestimated. ${ }^{[5]}$ Despite the significant health care burden associated with delirium, however, it is widely under-recognized, especially in the older population.

\section{RISK FACTORS}

The etiology of delirium is likely multifactorial and involves a complex relationship between predisposing and precipitating risk factors. Predisposing factors characterize a patient's susceptibility to developing delirium and are generally classified as non-modifiable. Precipitating factors are potentially modifiable iatrogenic insults that may trigger delirium (see Table 1). A patient with high baseline vulnerability such as advanced age, dementia, or multiple comorbid conditions may develop delirium following exposure to a relatively benign insult like a dose of a benzodiazepine. Conversely, multiple noxious insults may be required to trigger an episode of delirium in a patient with low baseline vulnerability. ${ }^{[19]}$ Several conflicting independent risk factors for delirium have been described in the literature; however, dementia has consistently been shown to be a prominent predictor across various clinical settings. ${ }^{[20]}$

Biomarkers that have been shown to have association with delirium include interleukin 8, cortisol, lactate, protean, acetylcholinesterase, and lower levels of somatostatin, Bendorphins, and neuron-specific enolase. ${ }^{[21]}$ To date, however, there is no agreed upon blood test that can be used to predict or diagnose delirium.

The dearth of evidence for the optimal treatment of delirium has led to a paradigm shift towards preventive efforts. 
Researchers have developed predictive risk models for systematic identification of at-risk patients on the basis of their clinical or environmental predisposition. ${ }^{[22,23]}$ Effective man- agement of modifiable risk factors is an integral component of a successful delirium prevention program. ${ }^{[10]}$

Table 1. Risk factors for delirium

\begin{tabular}{|c|c|}
\hline Predisposing (non-modifiable) & Precipitating (potentially modifiable) \\
\hline \multicolumn{2}{|l|}{ Demographic characteristics } \\
\hline - $\quad$ Age $>65$ years old, male sex & Drugs \\
\hline Cognitive status & - $\quad$ Sedative hypnotics, anticholinergics, narcotics, \\
\hline - $\quad$ Dementia & benzodiazepines, drug and alcohol withdrawal \\
\hline Functional status & Intercurrent Illness \\
\hline - Immobility, history of falls & - Infection, dehydration, trauma, shock, metabolic \\
\hline Sensory impairment & abnormalities, anemia, iatrogenic complications, \\
\hline - Visual, hearing & malnutrition \\
\hline Drugs & Surgery \\
\hline Alcohol abuse & - $\quad$ Orthopedic, cardiac, noncardiac \\
\hline - $\quad$ Polypharmacy & Acute neurologic diseases \\
\hline Comorbid conditions & - $\quad$ Stroke, meningitis, hemorrhage \\
\hline - $\quad$ Severe illness, stroke, neurologic disease, trauma, chronic & Environmental \\
\hline kidney and liver disease & - ICU admission, pain, physical restraints, catheters, \\
\hline \multicolumn{2}{|r|}{ emotional distress, sustained sleep deprivation } \\
\hline - $\quad$ Dehydration, malnutrition & \\
\hline
\end{tabular}

Note. ICU, intensive care unit. Source: Inouye, 2006. ${ }^{[5]}$

\section{DRUGS AND DELIRIUM}

Currently, there are no approved medications to prevent or treat delirium. Low doses of haloperidol, gabapentin, and donepezil have been researched with inconsistent results. ${ }^{[5,20,21]}$ Ramelteon administered nightly to older patients may provide protection against delirium, however larger studies are needed to confirm this finding. ${ }^{[24]}$ To prevent medication-induced delirium, it is prudent to avoid potentially high risk medications in older patients when possible. If a certain medication cannot be avoided or a safer alternative does not exist, it is best to use the smallest effective dose for the shortest period of time to limit patient exposure. A list of high-risk medications for older patients can be found in the Beer's Criteria or in the Start and the STOPP guidelines. ${ }^{[25,26]}$ Examples of high risk drug classes include anticholinergics, benzodiazepines, muscle relaxers, hypnotics, and opioids.

\subsection{Anticholinergics}

Anticholinergic medications have several useful indications; however, they also have an unfavorable side effect profile in older patients, including dry mouth, blurred vision, constipation, difficulty in urination, drowsiness, sedation, and impaired memory. Diphenhydramine is a first-generation antihistamine drug with sedating and strong anticholinergic properties. It is linked to delirium and other negative side effects in older patients. A study done in Yale-New Haven
Hospital showed that symptoms of delirium were much more likely to occur in a diphenhydramine-exposed group than in an unexposed group. Furthermore, the risk of cognitive decline was $70 \%$ higher in the diphenhydramine-exposed group. ${ }^{[21]}$ Potentially safer alternatives to diphenhydramine are less sedating second-generation antihistamines such as cetirizine and fexofenadine.

\subsection{Benzodiazepine}

Benzodiazepines have heterogeneous indications including seizures, alcohol withdrawal and anxiety with limited available alternatives. Adverse effects associated with this class of medications include drowsiness, confusion, dizziness, blurred vision, slurred speech, lack of coordination and respiratory depression. Except for necessary indications, it is best to limit exposure to benzodiazepines in the older person, especially when used for the treatment of insomnia, anxiety and agitation. ${ }^{[5]}$

A study conducted in post-surgical patients found a more pronounced association of long-acting benzodiazepines with delirium compared with shorter-acting benzodiazepines. ${ }^{\text {[23] }}$ Moreover, higher doses of benzodiazepine medications during a 24-hour period appear to be associated with an increased risk of delirium compared with lower doses. If benzodiazepines cannot be avoided, a suggestion is to use the lowest dose and shortest acting formulation possible to re- 
duce the risk of adverse effects such as delirium.

\subsection{Muscle relaxants}

Most muscle relaxants are poorly tolerated in older adults and should be avoided when possible due to anticholinergic side effects, sedation and risk of fractures. ${ }^{[25]}$ Furthermore, these drugs have not been proven to be superior to acetaminophen or non-steroidal anti-inflammatory drugs for acute low back pain. It is essential to balance a patient's intolerance with the aforementioned alternatives with the potential adverse effects from muscle relaxants. For the treatment of uncontrolled low back pain despite conservative approaches, these agents may be considered as an adjuvant therapy for a short term at the lowest effective dose. ${ }^{[27]}$

\subsection{Sedative hypnotics}

Non-benzodiazepine hypnotics including zolpidem, eszopiclone, and zaleplon have adverse effect profiles similar to benzodiazepines. These medications should be avoided in older patients due to the risk of delirium, falls and fractures. ${ }^{[25]}$ A meta-analysis performed on the risk versus benefit of sedative hypnotics showed that the total sleep time increased by a mean of 25.2 minutes only. As expected, side effects were more common with sedatives, including psychomotor events and daytime fatigue. The authors concluded that although the benefits were statistically significant, they were not clinically significant and that the risks outweigh the benefits of these medications. ${ }^{[25]}$

Ramelteon, a synthetic analogue of melatonin is associated with shortened sleep onset latency and no evidence of adverse cognitive effects. This agent may be considered as a potentially safer pharmacologic alternative for the treatment of insomnia in older patients. Moreover, the use of nonpharmacologic options such as quiet time and minimizing disturbances at night, having a proper awake-sleep cycle, and relaxing before bedtime is encouraged. ${ }^{[28]}$

\subsection{Opioids}

Preventing pain is not an easy process. Pain is difficult to treat in older patients. There is a fine line between treating pain adequately and over-treating patients so that side effects, such as delirium, occur and can become evident. Older patients have decreased drug clearance; therefore, a high dose (e.g., > $10 \mathrm{mg}$ of morphine) can have negative effects. On the other hand, inadequate treatment of pain is also a risk factor for delirium. ${ }^{[4]}$ It is important to know if the patient is opioid naïve or tolerant to help adjust medication doses. When treating pain, be cognizant of how the patient tolerates and reacts to the dose of medication. Alternating opioid and non-opioid pain medications can help decrease exposure, while staying ahead of the pain.

\section{RECOGNITION OF DELIRIUM}

Delirium is predominantly a clinical diagnosis and requires meticulous assessment of key features including disturbance in consciousness and impaired attention. ${ }^{[5]}$ Delirium has three subtypes based on psychomotor activity: hypoactive (lethargic, apathetic), hyperactive (restlessness, agitation), and a mix of both. ${ }^{[29]}$ Hypoactive delirium is the most common in older patients; it often goes unrecognized and usually is associated with a worse prognosis than the other motor subtypes. ${ }^{[23,30,31]}$ Patients presenting with mixed delirium can fluctuate between hypoactive and hyperactive manifestations. Early recognition is crucial because delirium may be the first sign of an underlying severe illness, and timely management may limit adverse outcomes. ${ }^{[5]}$ Routine screening of delirium in high-risk populations is feasible and recommended in clinical practice. ${ }^{[9]}$ The current standard for delirium diagnosis can be found in the Diagnostic and Statistical Manual of Mental Disorders, fifth edition (see Table 2).

\subsection{Differentiating delirium from dementia}

Diagnosing delirium can be challenging in the setting of advanced age, dementia, or concomitant psychiatric conditions. ${ }^{[32]}$ Silverstein and colleagues synthesized current research regarding delirium and dementia. They noted that several studies suggested a causative relationship between delirium and dementia and proposed the proverbial chicken or egg question in patients investigated. Dementia has recently been shown to be a high risk factor for delirium, especially in hospitalized, older patients. However, recent literature has hypothesized that some patients without dementia, developed delirium while hospitalized and subsequently showed some form of permanent cognitive impairment. ${ }^{[33]}$ Perioperative neurotoxicity and inflammation have been shown to cause a downward, long-lasting cognitive dysfunction. Furthermore, delirium superimposed on dementia can make the dementia worse $^{[29]}$ and can be an added challenge to the staff treating these patients. To adequately protect our vulnerable patients, diligence and persistence is required by the bedside clinician. This includes attention to the prevention, identification, investigation, and treatment of the cause of the delirium. The hallmark feature differentiating delirium from underlying dementia is inattention. Inattention, as described by Inouye, is the inability to focus, inability to sustain a conversation and the inability to follow commands.$^{[5]}$

For the nurse in the hospital setting, the task of differentiating delirium from dementia can be a challenge. The prevalence of dementia can be high as $89 \%$ in hospitalized patients. ${ }^{[34]}$ Unfortunately, delirium in patients with dementia is often not recognized owing to the similarities in presentation between delirium and dementia. To make matters worse, often, 
when the bedside clinician is aware of the history of dementia, almost all unusual activity of the patient is attributed to the dementia and no other investigation is done. ${ }^{[33]}$ Given a complex patient scenario like delirium with dementia, the probability of delirium should always be considered until proven otherwise. Therefore, it is imperative that all health care providers be able to distinguish the two presentations and act accordingly when delirium, or the risk of delirium, is suspect.

Delirium is diagnosed with a combination of measurable symptoms, including acute altered mental status, inattention, disorganization of thinking, and altered alertness. The onset of delirium is acute and fluctuates. This is in contrast with dementia, which progresses slowly and does not fluctuate. ${ }^{[35]}$ In addition, delirium's acute onset will likely be associated with a change in the patient's environment of physiology, such as hospitalization, surgery, or infection. The common symptom between dementia and delirium is disorganized thinking. Thus, knowledge of the patient's history is very important and key when attempting to differentiate between dementia and delirium. Family and caregivers can be a great source of information on baseline cognitive function.

Table 2. DSM-V criteria

When taking care of a hospitalized patient with dementia, the bedside clinician must have a high suspicion of the risk of delirium. Much of the recognition, prevention, investigation of source, and treatment of the patient with delirium is the same in a patient with dementia as in a patient without dementia.

\subsection{Recognition tools}

Several instruments have been developed for screening, diagnosis, or assessment of severity with varying sensitivities, specificities, and complexities based on the operational application of the DSM-IV diagnostic criteria. ${ }^{[33]}$ Common ex- amples include the CAM, 4AT, Nursing Delirium Screening Scale (Nu-DESC), and NEECHAM Confusion Scale. ${ }^{[4,36-38]}$ These instruments are mostly designed to be completed by nurses and incorporate information obtained from patient observation, caregivers, or the medical record. The CAM tool is standardized and widely accepted owing to its brevity and reliability (see Figure 1). It has shown a sensitivity of $94 \%$ to $100 \%$, specificity of $90 \%$ to $95 \%$, and high interrater reliability when administered by trained interviewers. ${ }^{\text {[4] Sev- }}$ eral laboratory tests have been investigated for possible use in evaluating delirium; however, these test are currently experimental and are not routinely used in clinical practice. ${ }^{[39]}$

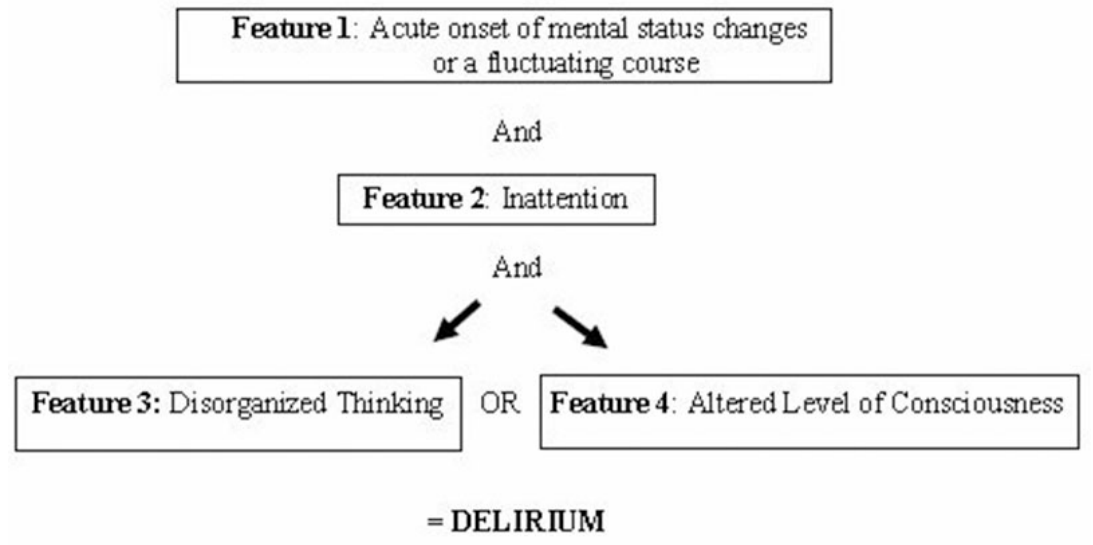

Figure 1. The CAM tool

Source: Inouye et al. $(1990)^{[4]}$ 
Once the diagnosis is established, initial assessment should focus on investigating potential causes of delirium to deliver targeted interventions to patients. A useful mnemonic for re- membering possible causes of delirium is I WATCH DEATH (see Table 3).

Table 3. Useful pneumonic to identify causes of delirium

\begin{tabular}{|ll|}
\hline I & Infections (urinary tract infection, pneumonia, encephalitis) \\
W & Withdrawal (alcohol, benzodiazepines, sedatives-hypnotics) \\
A & Acute metabolic (alcohol, benzodiazepines, sedatives-hypnotics) \\
T & Toxins, drugs (opiates, steroids, anticholinergics, psychotropics) \\
C & CNS pathology (stroke, tumor, seizures, hemorrhage, infection) \\
H & Hypoxia (anemia, pulmonary/cardiac failure, hypotension) \\
D & Deficiencies (thiamine [with alcohol abuse], B-12) \\
E & Endocrine (thyroid, hypo/hyperglycemia, adrenal insufficiency) \\
A & Acute vascular (shock, hypertensive encephalopathy) \\
T & Trauma (head injury, postoperative, falls) \\
H & Heavy metals \\
Note. & \\
\hline
\end{tabular}

\section{Management of Delirium}

As stated above, management of delirium is first, a concentrated effort in prevention. Prevention of delirium is key in avoiding negative side effects and outcomes. Adequate prevention strategies should be implemented upon recognition of a high-risk patient. Prevention methods are often multifactorial and multidisciplinary. Many hospitals are adapting innovative methods to recognize and combat this devastating syndrome. Once the high-risk patient is identified, strategies such as continued orientation, adequate nutrition, hydration and sleep, promoting mobility, limiting exposure to high risk medications and correcting sensory impairments are important to prevent delirium. These are recommendations from the Hospital Elder Life Program (HELP), which has many suggestions and resources for caring for the elderly in the hospital. ${ }^{[41]}$

Enhancing delirium awareness is essential to combat delirium. When the health care team is aware and vigilant of signs of delirium, actions can be taken with the first sign of delirium, which, in turn, can prevent a full-blown delirious state and the sequelae afterwards. Studies have shown that prevention of delirium in postsurgical patients is improved with early involvement of a geriatric team. ${ }^{[21]}$ Additional studies have shown decreases in delirium in postsurgical patients who have early sleep protocols and appropriate pain management. ${ }^{[42]}$ Despite adequate prevention in the at-risk patient population, patients may still develop delirium. When prevention switches to treatment of delirium, the prevention strategies should continue, with the addition of safety interventions and investigation into possible causes of the delirium.

Published by Sciedu Press
Evidence-based, best practices for management recommended by Tullman et al. are the same as prevention strategies but include safety and treatment measures. ${ }^{[43]}$ One recommendation found in many articles is to consult with a geriatric specialist. This could be done either for prevention or treatment and would depend on the risk of the patient for delirium. Patients at higher risk include patients with dementia, patients with extended intensive care unit stays, and postoperative patients, especially those with hip fracture repair and coronary artery bypass grafting.

Recommendations to reduce risk factors include minimizing excessive medication administration as much as possible and, when possible, avoiding high-risk medications. ${ }^{[43]}$ Treating any sign that is a suspicious cause of delirium is a recommendation and can be decided upon with a good-quality history and physical assessment. Treating infection, dehydration, and electrolyte disturbances are beyond the scope of a bedside nurse practice, but can easily be discussed and recommended to the treating provider such as the attending physician or advanced practice provider. Many of the suggestions or recommendations for treatment require a multidisciplinary approach. The nurse may be the first to recognize the possible causes of the delirium. For example, pain control is recommended to treat and prevent delirium. If the nurse recognizes or suspects pain in the elderly patient, both nonpharmacological and pharmacological interventions can be tried depending on the orders. If no orders are present, collaboration with the provider to obtain the needed order would be the first step in controlling the patient's pain. Other helpful strategies to prevent and treat delirium can include additional oxygen, blood pressure support, and mobilization. ${ }^{[43]}$ Col- 
laborating with the providers, family, and nursing assistants to ensure adequate nutrition, sensory aids, and toileting are important factors in the prevention and treatment of delirium.

A therapeutic environment is recommended as well by best practices. ${ }^{[43]}$ Reassurance and orientation for the patient can be very helpful and can be accomplished by all professionals, sitters and nursing assistants as well as family members. Low stimulation and adequate sleep should be promoted throughout the patient's hospital stay. Avoiding urinary catheters and restraints are recommended if at all possible. Although once commonplace, these two interventions have been shown to be a common trigger for delirium and may also do physical harm to the patient.

Another factor for management that is highly recommended in multiple sources is mobility. The bedside nurse is usually the first to evaluate patients for mobility. When the patient is not able to mobilize independently, the nurse can request physical therapy evaluation and then collaborate with physical therapy for the patient's needs. Recommendations from the therapist may include mobility devices, such as walkers or canes, and would also require collaboration with the health care provider as well as a case manager to obtain recommended devices.

Nonpharmacological methods of prevention simply involve being more attuned to high-risk patients. When high-risk patients are identified, special considerations should be taken to ensure proper cognitive function is intact. Table 4 lists different measures to help high-risk patients.

Table 4. Methods to prevent delirium in high-risk patients

Reorient patients as needed to person, time, and place
Provide a comfortable physical environment
- Example: clocks, natural light, stimulate day/night, quiet times for rest
Avoid the use of hypnotics. Use non-pharmacological measures
Provide activities to stimulate the brain
- Example: crossword puzzles, word games, books to read, conversation
Provide eyeglasses, hearing aids, and amplifiers for sensory orientation
Monitor for signs of infections, lab abnormalities, fluid imbalance
• Example: offer a variety of healthy foods and snacks; avoid caffeine and extra sugar
Note. Source: Inouye, $2004 .^{[44]}$

\section{INTERVENTION AND TREATMENT}

The highest priority with delirium management is prevention. However, when delirium does develop, then prevention switches to identification and treating the underlying cause of delirium as promptly as possible. This would include infection and fluid and electrolyte imbalance which are two of the most common causes of delirium. Pharmacological treatment should be avoided unless necessary, such as when a patient's agitation is making an unsafe environment for the patient, family members, and health care providers. ${ }^{[4]}$

Delirium can actually be a manifestation of a possibly life threatening insult to the patient. Therefore, treatment of delirium involves treatment of the underlying cause. Infection is a common cause for delirium and two of the most common causes are pneumonia and urinary tract infection. The bedside clinician should be aware that older people sometimes do not have a typical immune response to infection and thus, delirium may be the only presentation seen. ${ }^{[5]}$ Diagnostic testing such as a chest $\mathrm{x}$-ray or a urine culture would be the easiest diagnostics to confirm suspicion of these infections. Appropriate antibiotics could then be ordered by the health care provider. Delirium caused by electrolyte imbalances is 14 easily diagnosed with common lab work. Appropriate electrolyte replacement or treatment can then be ordered by the health care provider and initiated by the bedside clinician.

One of the first interventions is to ensure that all team members are aware of the patient's change in mental status. Other team interventions include frequent reorientation, fall prevention measures, bed and chair alarms, hourly mental status reassessment, frequent toileting, increasing vital sign assessment, and monitoring blood glucose and oxygen saturation levels. For suspicion or diagnosis of dehydration as a cause of delirium, replacement of volume by intravenous fluids or encouragement of intake is appropriate when patients are not confused. Reviewing the patient's medication profile and removing potential harmful medication can be done with partnership with pharmacy and prescriber(s). Another intervention is to increase mobility as well as to use sensory aids in patients with difficulty seeing or hearing. To address these concerns, physical therapy could be consulted for mobility concerns, and hearing aids or amplifiers, glasses, and communication tools should be provided to appropriate patients. Other useful tools are to have familiar objects in the patient's rooms as well as objects that can occupy the patients' time, 
such as puzzles and stuffed animals. ${ }^{[43]}$

\section{Conclusion}

In conclusion, prevention of delirium is key to avoiding negative side effects and outcomes. One key element of delirium to remember, is that the presentation can vary between periods of confusion and clarity. Optimizing interventions during periods of clarity can be both safe and beneficial to the patient. Certain medications should be avoided or appropriately spaced out to prevent delirium. The causes of delirium can be multifactorial and may impact both outcomes and costs for hospitalized patients. These outcomes include length of stay, avoidable readmissions, increased morbidity and mortality, and institutionalization. Many hospitals are adapting innovative methods to combat the devastating syndrome of delirium. Health care facilities are also incorporating the use of screening tools. These tools need to be chosen according to each institution's situation and the usefulness and reliability of the tool. When the health care team is aware of the signs of delirium, vigilant actions can be taken at the first sign of any significant change in the patient. These patients and families need additional support with prevention and treatment strategies.

\section{REFERENCES}

[1] Khan AR, Kahn D, Bourgeois JA. Delirium: sifting through the confusion. Curr Psychiatry Rep. 2009; 11(3): 226-234. http: //dx.doi.org/10.1007/s11920-009-0035-8

[2] Frederiks JA. Inflammation of the mind on the 300th anniversary of Gerard Van Swieten. J Hist Neurosci. 2000; 9(3): 307-310. http:// dx.doi.org/10.1076/0964-704X (200012) 9:3;1-\#;FT307

[3] Francis J. Historical overview of investigations into delirium. Prim Psychiatry. 2004; 11(11): 31-35.

[4] Inouye SK, Van Dyck CH, Alessi CA, et al. Clarifying confusion: the confusion assessment method. A new method for detection of delirium. Ann Intern Med. 1990; 113(12): 941-948. http://dx.doi.org/10.7326/0003-4819-113-12-941

[5] Inouye SK. Delirium in older persons. N Engl J Med. 2006; 354(11): 1157-1165. http://dx.doi.org/10.1056/NEJMra052321

[6] Van der Mast RC. Pathophysiology of delirium. J Geriatr Psychiatry Neurol. 1998 Fall; 11(3): 138-45. PMid:9894732 http: //dx.doi.org/10.1177/089198879801100304

[7] A Profile of Older Americans: 2001. Washington (DC): Administration on Aging, US Department of Health and Human Services. 2002.

[8] Leslie DL, Marcantonio ER, Zhang Y, et al. One-year health care costs associated with delirium in the elderly population. Arch Intern Med. 2008; 168(1): 27-32. http://dx.doi.org/10.1001/arc hinternmed.2007.4

[9] Grover S, Kate N. Assessment scales for delirium: A review. World J Psychiatry. 2012; 2(4): 58-70. http://dx.doi .org/10.5498/w jp.v2.i4.58

[10] Inouye SK, Bogardus ST Jr, Charpentier PA, et al. A multicomponent intervention to prevent delirium in hospitalized older patients. N Engl J Med. 1999; 340(9): 669-676. http://dx.doi .org/10.1056/N EJM199903043400901

[11] Girard TD, Pandharipande PP, Carson SS, et al. Feasibility, efficacy, and safety of antipsychotics for intensive care unit delirium: the MIND randomized, placebo-controlled trial. Crit Care Med. 2010; 38(2): 428-437. http://dx.doi.org/10.1097/CCM.0b0 13 e3181c58715

[12] Bruce AJ, Ritchie CW, Blizard R, et al. The incidence of delirium associated with orthopedic surgery: a meta-analytic review. Int Psychogeriatr. 2007; 19(02): 197-214. http://dx.doi.org/10.1017 /S104161020600425X

Published by Sciedu Press
[13] Pisani MA, McNicoll L, Inouye SK. Cognitive impairment in the intensive care unit. Clin Chest Med. 2003; 24(4): 727-737. http: //dx.doi.org/10.1016/S0272-5231(03)00092-3

[14] Folstein MF, Bassett SS, Romanoski AJ, et al. The epidemiology of delirium in the community: the Eastern Baltimore Mental Health Survey. Int Psychogeriatr. 1991; 3(2): 169-176. http: //dx.doi.org/10.1017/S1041610291000637

[15] Inouye SK, Rushing JT, Foreman MD, et al. Does delirium contribute to poor hospital outcomes? A three-site epidemiologic study. J Gen Intern Med. 1998; 13(4): 234-242. http://dx.doi.org/10.1046 /j.1525-1497.1998.00073.x

[16] Siddiqi N, House AO, Holmes JD. Occurrence and outcome of delirium in medical in-patients: A systematic literature review. Age Ageing. 2006; 35(4): 350-364. http://dx. doi .org/10.1093/agein g/af 1005

[17] Inouye SK, Schlesinger MJ, Lydon TJ. Delirium: A symptom of how hospital care is failing older persons and a window to improve quality of hospital care. Am J Med. 1999; 106(5): 565-573. http://dx.doi.org/10.1016/S0002-9343(99)00070-4

[18] Leslie DL, Zhang Y, Holford TR, et al. Premature death associated with delirium at 1-year follow-up. Arch Intern Med. 2005; 165(14): 1657-1662. http://dx.doi.org/10.1001/archinte. 165.14 .1657

[19] Inouye SK. Delirium in hospitalized older patients. Clin Geriatr Med. 1998; 14(4): 745-764. PMid:9799477

[20] Inouye SK, Charpentier PA. Precipitating factors for delirium in hospitalized elderly persons. Predictive model and interrelationship with baseline vulnerability. JAMA. 1996; 275(11): 852-857. http://dx.doi.org/10.1001/jama.1996.03530350034031

[21] Khan BA, Zawahiri M, Campbell NL, et al. Delirium in hospitalized patients: implications of current evidence on clinical practice and future avenues for research-a systematic evidence review. J Hosp Med 2012; 7(7): 580-589. http://dx.doi.org/10.1002/jhm. 1949

[22] Elie M, Cole MG, Primeau FJ, et al. Delirium risk factors in elderly hospitalized patients. J Gen Intern Med. 1998; 13(3): 204-212. http://dx.doi.org/10.1046/j.1525-1497.1998.00047.x

[23] O'Keeffe ST, Lavan JN. Clinical significance of delirium subtype in older people. Age Ageing. 1999; 28(2): 115-119. http://dx.doi . org/10.1093/ageing/28.2.115

[24] Hatta K, et al. Preventive effects of ramelteon on delirium-A randomized placebo-controlled trial. JAMA Psychiatry. 2014; 71(4): 
397-403. PMid:24554232 http://dx.doi.org/10.1001/jamap sychiatry.2013.3320

[25] The American Geriatrics Society 2012 Beers Criteria Update Expert Panel. American Geriatrics Society Updated Beers Criteria for Potentially Inappropriate Medication Use in Older Adults. J Am Geriatr Soc. 2012; 60: 616-631. PMid:22376048

[26] Gallagher P, O’Mahony D. STOPP (Screening Tool of Older Persons' potentially inappropriate Prescriptions): application to acutely ill elderly patients and comparison with Beers' criteria. Age Ageing. 2008; 37(6): 673-679. http://dx.doi.org/10.1093/ageing/ afn 197

[27] Chou R, Qaseem A, Snow V, et al. Diagnosis and treatment of low back pain: A joint clinical practice guideline from the american college of physicians and the american pain society. Ann Intern Med. 2007; 147(7): 478-91. http://dx.doi.org/10.7326/0003-481 9-147-7-200710020-00006

[28] Roth T, Seiden D, Wang-Weigand S, et al. A 2-night, 3-period, crossover study of ramelteon's efficacy and safety in older adults with chronic insomnia. Curr Med Res Opin. 2007; 23: 1005 14. PMid:17519067 http://dx.doi.org/10.1185/030079907 $\mathrm{X} 178874$

[29] Liptzin B, Levkoff SE. An empirical study of delirium subtypes. $\mathrm{Br}$ J Psychiatry. 1992; 161(6): 843-845. http://dx.doi .org/10.11 92/bjp.161.6.843

[30] Robinson TN, Raeburn CD, Tran ZV, et al. Motor subtypes of postoperative delirium in older adults. Arch Surg. 2011; 146(3): 295-300. http://dx.doi.org/10.1001/archsurg. 2011.14

[31] Inouye SK, Foreman MD, Mion LC, et al. Jr Nurses' recognition of delirium and its symptoms: comparison of nurse and researcher ratings. Arch Intern Med. 2001; 161(20): 2467-2473. http://dx.doi.org/10.1001/archinte.161.20.2467

[32] Fick DM, Mion LC. Delirium superimposed on dementia. Am J Nurs. 2008; 108(1): 52-60. http://dx.doi.org/10.1097/01. NAJ . 00 $00304476.80530 .7 \mathrm{~d}$

[33] Silverstein JH, Deiner SG. Perioperative delirium and its relationship to dementia. Prog Neuropsychopharmacol Biol Psychiatry. 2013; 43: 108-115. http://dx.doi.org/10.1016/j.pnpbp.2012.11 .005

[34] Fong TG, Tulebaev SR, Inouye SK. Delirium in elderly adults: diagnosis, prevention and treatment. Nat Rev Neurol. 2009; 5(4): 210-
220. PMid:19347026 http://dx.doi.org/10.1038/nrneurol. 2009.24

[35] Fong TG, Davis D, Growdon ME, et al. The interface between delirium and dementia in elderly adults. Lancet Neurol. 2015; 14(8): 823832. http://dx.doi.org/10.1016/S1474-4422(15)00101-5

[36] Bellelli G, Morandi A, Davis DH, et al. Validation of the 4AT, a new instrument for rapid delirium screening: a study in 234 hospitalized older people. Age Ageing. 2014; 43(4): 496-502. http://dx.doi.org/10.1093/ageing/afu021

[37] Gaudreau JD, Gagnon P, Harel F, et al. Fast, systematic and continuous delirium assessment in hospitalized patients: the nursing delirium screening scale. J Pain Symptom Manage. 2005; 29(4): 368-375. ht tp://dx.doi.org/10.1016/j.jpainsymman.2004.07.009

[38] Neelon VJ, Champagne MT, Carlson JR, et al. The NEECHAM Confusion Scale: construction, validation, and clinical testing. Nurs Res. 1996; 45(6): 324-330. http://dx.doi.org/10.1097/000 06199-199611000-00002

[39] Trzepacz P, Breitbart W, Franklin J, et al. Practice guideline for the treatment of patients with delirium. American Psychiatric Association; 2010. Available from: http://psychiatryonline.org/pb/assets/raw/sitew ide/practice_guidelines/guidelines/delirium.pdf

[40] I WATCH DEATH Pneumonic for delirium causes. Nursing best practice guidelines [cited 2015 May]. Available from: http://pda. rnao.ca/content/causes-delirium

[41] Reuben DB, Inouye SK, Bogardus ST Jr, et al. The Hospital Elder Life Program: a model of care to prevent cognitive and functional decline in older hospitalized patients. J Am Geriatr Soc. 2000; 48(12): 1697-1706. http://dx.doi.org/10.1111/j.1532-5415.2000 .tb03885.x

[42] Reston JT, Schoelles KM. In-facility delirium prevention programs as a patient safety strategy: a systematic review. Ann Intern Med. 2013; 5(158): 375-80. http://dx.doi.org/10.7326/0003-481 9-158-5-201303051-00003

[43] Tullman DF, Fletch K, Foreman MD. Delirium: Evidence-Based Geriatric Nursing Protocols for Best Practice. New York: Springer Publishing Company; 2012: 186-199.

[44] Inouye SK. A practical program for preventing delirium in hospitalized elderly patients. Cleve Clin J Med. 2004; 71(11): 890-896. http://dx.doi.org/10.3949/ccjm.71.11.890 